\title{
Light induces chromatin modification in cells of the mammalian circadian clock
}

\author{
Claudia Crosio ${ }^{1}$, Nicolas Cermakian ${ }^{1}$, C. David Allis ${ }^{2}$ and Paolo Sassone-Corsi ${ }^{1}$ \\ ${ }^{1}$ Institut de Génétique et de Biologie Moléculaire et Cellulaire, CNRS - INSERM - Université Louis Pasteur, 1 rue Laurent Fries, Illkirch-Strasbourg 67404, France \\ 2 University of Virginia H.S.C., Department of Biochemistry and Molecular Genetics, Box 800733, Charlottesville, Virginia 22908-0733, USA \\ The first two authors contributed equally to this work \\ Correspondence should be addressed to P.S.-C. (paolosc@igbmc.u-strasbg.fr)
}

The mammalian circadian clock resides in neurons of the hypothalamic suprachiasmatic nucleus (SCN). Light entrains phase resetting of the clock using the retino-hypothalamic tract, via release of glutamate. Nighttime light exposure causes rapid, transient induction of clock and immediate-early genes implicated in phase-shifting the pacemaker. Here we show that a nighttime light pulse caused phosphorylation of Ser10 in histone H3's tail, in SCN clock cells. The effect of light was specific, and the kinetics of $\mathrm{H} 3$ phosphorylation were characteristic of the early response, paralleling c-fos and Per1 induction. Using fos-lacZ transgenic mice, we found that H3 phosphorylation and Fos induction occurRed in the same SCN neurons. Systemic treatment with the GABA receptor agonist baclofen prevented light-induced c-fos and Perl expression and H3 phosphorylation, indicating that one signaling pathway governs both events. Our results suggest that dynamic chromatin remodeling in the SCN occurs in response to a physiological stimulus in vivo.

The central mammalian circadian clock is located in neurons of the hypothalamic suprachiasmatic nucleus ${ }^{1-3}$. The pacemaker activity of the SCN is autonomous, as it functions in constant darkness and in the absence of external light stimuli ${ }^{1-3}$; however, it can be entrained by environmental light-dark cycles ${ }^{4}$. In rodents, at least three neural pathways link the retina to the SCN: first, the direct, monosynaptic retino-hypothalamic tract (RHT), whose main neurotransmitters seem to be excitatory amino acids such as glutamate ${ }^{5,6}$; second, the indirect multisynaptic projection from the intergeniculate leaflet (IGL), which acts via the release of the protein neurotransmitter neuropeptide $\mathrm{Y}$ and the inhibitory neurotransmitter $\gamma$-aminobutyric acid (GABA) ${ }^{7,8}$; third, an indirect pathway arising from the dorsal and median raphe nucleus, in which serotonin is the main neurotransmitter ${ }^{9}$. Although the RHT alone seems necessary and sufficient to mediate light entrainment ${ }^{10}$, interference with the IGL and/or dorsal raphe nucleus transmission $^{11}$ can modify the response of the SCN to light.

In mammals kept in darkness, a light pulse during the subjective night (that is, the time of the day corresponding to the dark period in a normal light-dark cycle) causes phase-shifting of the $\mathrm{SCN}$-controlled rhythms ${ }^{4}$. The phase-shift stimulated by light triggers gene expression within the SCN, including the rapid and transient activation of clock genes, such as Per1 and Per2 (refs. 12, 13) and of immediate early genes (IEGs), such as c-fos, fos-B, jun-B, nur77 and zif268 (refs. 14, 15). These changes in gene expression have been implicated in phase-shifting the pacemaker ${ }^{16,17}$. Although the photic regulation of c-fos expression in the SCN has been extensively studied, the pivotal events enabling dynamic regulation at the chromosomal level are not yet known.

Light pulses induce activation of the mitogen activated protein kinase (MAPK) ${ }^{18}$, and thus possibly modulate the physiological function of substrates within the SCN. In particular, light pulses induce phosphorylation of transcription factor CREB (cAMP response-element binding protein) on serine 133 (ref. 19), the essential phospho-acceptor site that enables the recruiting of the co-activator CBP, a protein with histone acetyltransferase (HAT) activity ${ }^{20}$. Factors of the CREB family are involved in circadian rhythmicity both in Drosophila ${ }^{21}$ and mammals ${ }^{22}$.

Modifications occurring on histone tails are primary events that contribute to the dynamic process of chromatin remodeling, an essential step in transcriptional regulation ${ }^{23}$. Both chromatin remodeling factors and covalent histone modifications facilitate access of transcription factors to chromatin, and regulate expression of a wide range of genes ${ }^{24}$. A number of post-translational modifications occur on histone tails (for review, see ref. 23). Among these, inducible phosphorylation at serine 10 of the histone $\mathrm{H} 3 \mathrm{~N}$-terminal tail in response to a mitogenic stimulus represents the best characterized link between activation of a signal transduction pathway and chromatin modification. Phosphorylation occurs in a subset of histone $\mathrm{H} 3$ molecules in a rapid and transient manner, paralleling the induction profile of early response genes $^{25,26}$. We previously identified the MAPK-activated RSK2 kinase as a candidate for epidermal growth factor (EGF)-induced phosphorylation of H3 (ref. 25). RSK2 is also involved in EGFinduced CREB phosphorylation and c-fos expression in fibroblasts $^{27}$, establishing a direct link between signaling to chromatin and induction of IEGs. Finally, phosphorylation on Ser10 greatly enhances the subsequent HAT-mediated acetylation on the closely located Lys 14 on the $\mathrm{H} 3$ tail, underscoring the primary importance of phosphorylation ${ }^{28}$. Our knowledge of histone modifications is mostly based on studies in cell culture systems; histone modification function in vivo is basically unexplored. 
Fig. 1. Time- and light-dependent phosphorylation of histone $\mathrm{H3}$ in the suprachiasmatic nucleus. (a) Immunofluorescence on mouse $\mathrm{SCN}$ cryosections with the P-H3 specific antibody. Mice were dissected at four different circadian times (CT followed by the number of hours after the beginning of the subjective day), after a 15-min light pulse and $15 \mathrm{~min}$ in the dark (light) or at equivalent times but omitting the pulse (basal). (b) Q uantification of the P-H3 signal in the SCN at CT9 and CT21, after a light pulse (light) or not after a light pulse (basal). The data shown are a compilation of several independent experiments, as indicated. The increase in P-H 3 signal upon light stimulation at CT21 is statistically significant (Student's t-test, $p<0.01$ ). (c) Confocal microscopy analysis of independent $\mathrm{SCN}$ cells under basal conditions (top) or after a light pulse (middle and bottom). N uclear signal in light-stimulated mice varies from low staining with punctated pattern (middle) to intense and widespread staining (bottom).

Here we report that the photic stimulus has previously unrecognized profound effects on chromatin remodeling in non-proliferating neurons. A light pulse applied to mice during the subjective night induced the phosphorylation of the histone $\mathrm{H} 3$ in the ventrolateral SCN, but not in the pineal gland or retina. This transient modification occurred with early response kinetics, paralleling the induction of the c-fos gene. Moreover, $\mathrm{H} 3$ phosphorylation and gene induction were blunted by the $\mathrm{GABA}_{\mathrm{B}}$ receptor agonist baclofen, which counteracts light effects on the $\mathrm{SCN}^{29,30}$. Our results provide in vivo evidence of chromatin remodeling in the vertebrate nervous system in response to an external stimulus.

\section{RESULTS}

Light-inducible histone $\mathrm{H} 3$ phosphorylation in the SCN

Two groups of mice were placed for 2 weeks on a 12-12 hour light-dark (LD) cycle and then transferred to constant darkness (DD) for 4 days. At different circadian times (CT; CT0 corresponds to lights on), one group of mice was directly killed, whereas the second group was exposed to light for 15 minutes and then returned to darkness for 15 additional minutes before dissection. Phosphorylation was determined by immunofluorescence in whole-brain cryosections using a specific antibody directed against a phosphorylated Ser $10 \mathrm{H} 3$ peptide (P-H3; ref. 31). There was no spontaneous phosphorylation of $\mathrm{H} 3$ in the $\mathrm{SCN}$ at any time of the circadian cycle (Fig. 1a). A light pulse induces prominent $\mathrm{H} 3$ phosphorylation in the SCN, though only during the subjective night (when light causes phase-shift of the clock; for example, a delay at CT15 and an advance at CT21 $)^{4}$, and densitometric analy- a

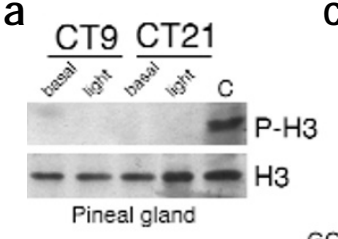

b

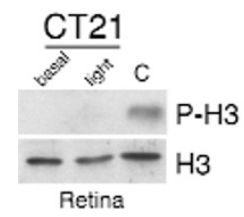

a

b

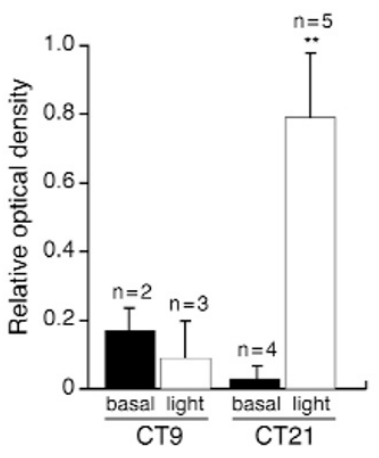

CT3

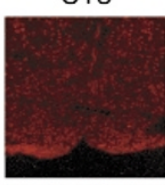

light
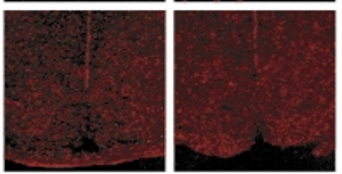

CT9

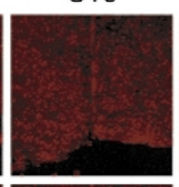

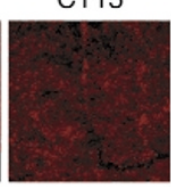
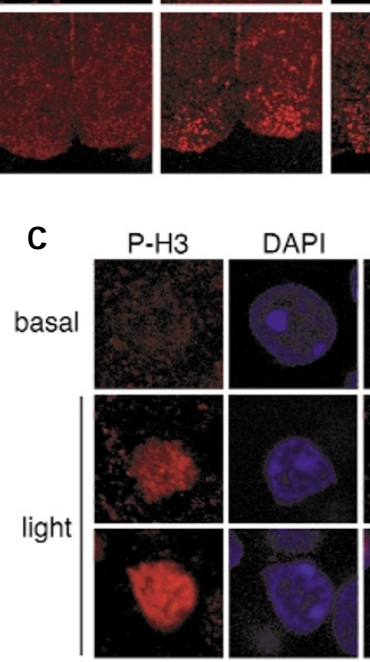

CT21

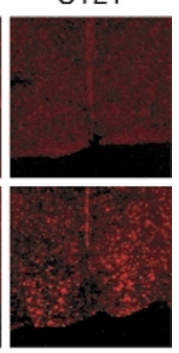

sis confirmed that the photic stimulation was statistically significant (Fig. 1b). No H3 phosphorylation was present in other brain areas. The SCN ventrolateral region, an area that displays maximal induction of c-fos in response to light ${ }^{14}$, displayed maximal H3 phosphorylation. Therefore, as for immediate early gene expression, H3 phosphorylation was induced by retinal illumination and was gated by the circadian clock.

Confocal analysis of SCN sections from mice light-stimulated at CT2 1 showed a nuclear staining pattern specific for the euchromatin (Fig. 1c). The staining, as previously observed in EGF-induced fibroblasts ${ }^{25}$, was not uniform over the nucleus, although it appeared more diffused as compared to the nuclear speckles observed in fibroblasts. This may be ascribed to the distinct experimental settings, as well as to the diverse signaling pathways involved; in our previous study, growth factors led to cell proliferation $^{25}$, whereas here light elicited a dynamic response in non-proliferating neurons.

\section{No H3 phosphorylation in retina and pineal gland}

In mammals, the $\mathrm{SCN}$ receives photic information from the retina, which also contains a circadian pacemaker ${ }^{3}$. Circadian timing information is then relayed from the SCN to the pineal gland, thereby controlling the rhythmic release of the hormone melatonin $^{1,3}$. We thus studied the consequence of photic stimulation on $\mathrm{H} 3$ phosphorylation in these areas. Western blot analyses were

Fig. 2. Photic stimulation did not induce $\mathrm{H} 3$ phosphorylation in the pineal gland and in the retina. Western blot analyses were performed on pineal gland (a) isolated before (basal) and after a 15-min light pulse followed by 15-min in the dark (light) at CT 9 and CT21, and on retina (b) isolated before and after a light pulse at CT21. Control of H 3 phosphorylation in EGF-stimulated mouse fibrolablasts, third lane (C). (c) Immunohistochemistry with $\mathrm{P}-\mathrm{H} 3$ antibodies on retina sections. Mice were dissected at CT21 before or after a light pulse. $\mathrm{N}$ o induction of histone $\mathrm{H} 3$ phosphorylation is detected. Use of P-CREB antibodies as control for presence of phosphoproteins shows a signal in the inner nuclear layer (IN L). GCL, ganglion cell layer; O N L, outer nuclear layer. 
a

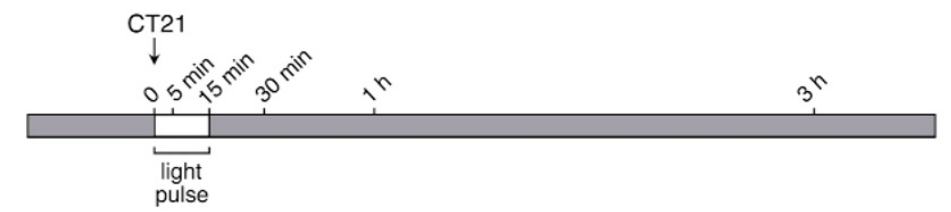

b

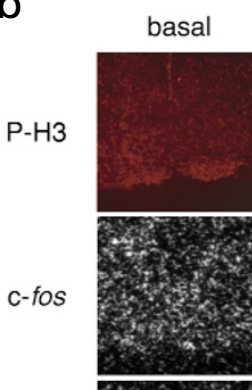

basal
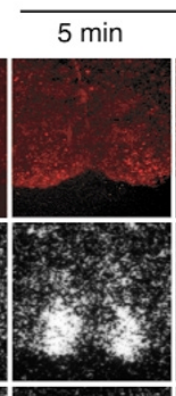

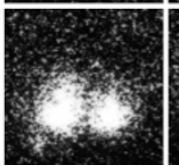

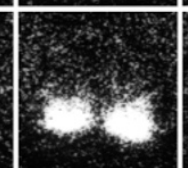

$15 \mathrm{~min}$

Light-pulsed

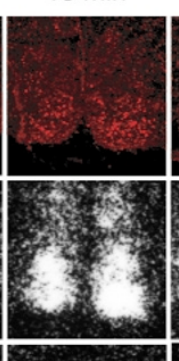

$30 \mathrm{~min}$
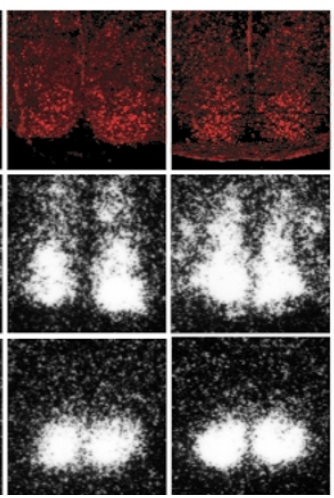
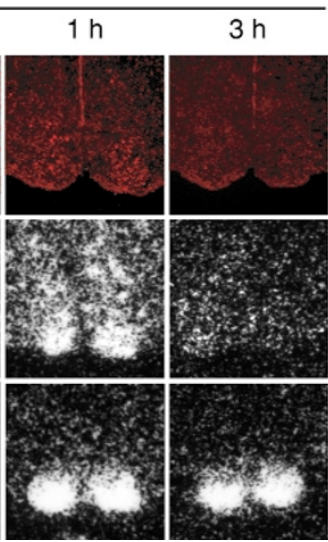

C

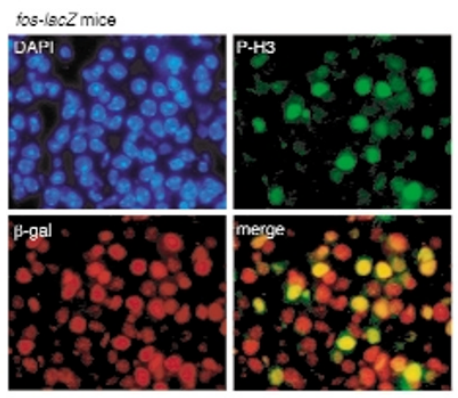

d

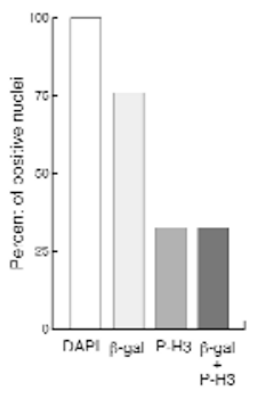

Fig. 3. Timing correlation of histone $\mathrm{H} 3$ phosphorylation and c-fos induction in the SCN . (a) Time course experiment presented in (b). Mice, entrained on a light-dark cycle and then placed in constant darkness for five days, were given a light-pulse at CT21. Mice were dissected after 5 or 15 min of lighting, or at different times after a 15-min light pulse. Brain cryosections were processed for either immunohistochemistry with P-H 3 specific antibody or in situ hybridization with c-fos or VIP probes. (c) Immunofluorescence on SC N cryosections (enlarged) from fos-lacZ transgenic mice. Double labeling with P-H3 and $\beta$-galactosidase antibodies of a single cryosection is shown, along with a combination of the P-H3 and $\beta$-gal images (merge). Mice were given a light-pulse at CT21 as described in (a) and dissected after two hours. (d) Q uantification of the data in (c). N eurons with the different stainings were counted and plotted as a percentage of the total number of neurons in the area (as assessed with DAPI). The procedure was repeated for several independent experiments, with analogous results.

done on pineal gland extracts during a normal light-dark cycle (data not shown), and after a light pulse at CT9 and CT21 (Fig. 2a). In both cases, no $\mathrm{H} 3$ phosphorylation was detectable. In eye samples collected at CT21, following or not following a light pulse, no $\mathrm{H} 3$ phosphorylation was observed (Fig. 2b). Also in retinal sections from mice stimulated or not stimulated with light at CT21 (Fig. 2c), CT3, CT9 and CT15 (data not shown), no signal was present, although control CREB phosphorylation was detected in the inner nuclear and ganglion cell layers (Fig. 2c). Thus, light-induced $\mathrm{H} 3$ phosphorylation seemed to be restricted to the cells of the central clock. These results stressed the specificity of SCN cells as receptors of the light signal, and of their capability to transduce the signal into a selected phosphorylation of one histone tail.

\section{Coupling H3 phosphorylation with c-fos expression}

To study the time course of $\mathrm{H} 3$ phosphorylation after photic induction, we stimulated animals with light for 15 minutes and returned them to the dark for various lengths of time before dissection. Other mice were exposed to light for five minutes and then killed immediately (Fig. 3a). We used serial sections from individual mice to probe c-fos gene expression and $\mathrm{H} 3$ phosphorylation in parallel. Expression of VIP was used as a control ${ }^{32}$. As for c-fos inducibility ${ }^{14}$, short light pulses were sufficient to induce $\mathrm{H} 3$ phosphorylation. Histone $\mathrm{H} 3$ phosphorylation peaks 30 minutes after the onset of light, whereas three hours later it is no longer detectable. Thus, the timing of $\mathrm{H} 3$ phosphorylation closely coincides with the early gene response (Fig. 3b).

To verify that induction of $\mathrm{H} 3$ phosphorylation and early gene expression occur concomitantly in the same SCN neurons in response to the light stimulus, we used transgenic fos-lac $Z$ mice ${ }^{33}$.
In these mice, $\beta$-galactosidase ( $\beta$-gal) expression driven by the c-fos promoter was inducible by a light-pulse at CT21, during the subjective night ${ }^{33}$. Nuclei positive for $\mathrm{H} 3$ phosphorylation, but not for $\beta$-gal, were detected one hour after the CT2 1 light pulse (data not shown). Two hours after the light pulse, $\beta$-galpositive and double-labeled nuclei were visible (Fig. 3c). Almost half of $\beta$-gal-positive nuclei (Cy3 labeled) also exhibited P-H3 staining (FITC labeled), whereas all $\mathrm{P}-\mathrm{H} 3$ positive nuclei were also stained with the anti- $\beta$-gal antibody (Fig. $3 \mathrm{~d}$ ). These data provide strong evidence that the two events occur concurrently in a large subset of SCN neurons.

\section{Baclofen blocks light-induced H3 phosphorylation}

The administration of the $\mathrm{GABA}_{\mathrm{B}}$ receptor agonist baclofen is known to inhibit phase-shifts induced by light during the subjective night, as well as c-fos gene expression ${ }^{29,30}$. We thus considered whether a pharmacological treatment modulating light-dependent effects on the clock would also interfere with the signaling routes required for $\mathrm{H} 3$ phosphorylation. Mice, entrained on a L12-D12 cycle for two weeks and housed in DD for four days, were injected intraperitoneally with baclofen or a saline solution at CT3, CT9, CT15 or CT21. After 10 minutes, animals were exposed to a 15-minute light pulse, and then placed in the dark for 15 minutes before dissection. We then analyzed gene expression and histone $\mathrm{H} 3$ phosphorylation in the SCN of these mice (Fig. 4).

Baclofen alone had no effect on IEGs or clock gene expression in the SCN at any time. On the other hand, baclofen strongly reduced c-fos induction in response to light at CT15 and CT21. A similar effect was observed on the light-induced expression of clock gene Per1. Transcript levels of Per1 oscillate during the day, 


\section{articles}
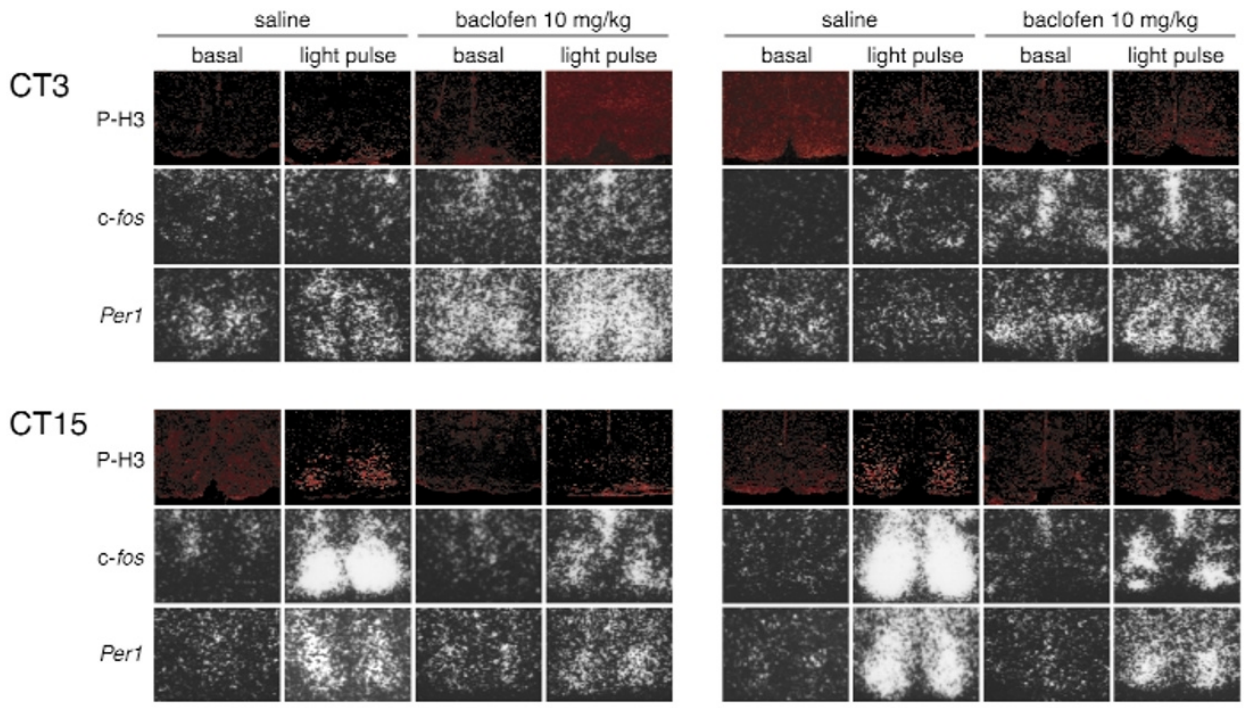

CT21

Fig. 4. Baclofen inhibits light-induced phosphorylation of histone $H 3$ and induction of $c$-fos and Perl gene expression in the SC N . Mice entrained as described in Fig. 1 were injected with saline solution or baclofen $(10 \mathrm{mg} / \mathrm{kg}) 10 \mathrm{~min}$ before a light pulse at CT3, CT9, CT15 and CT21. Brain cryosections were processed for either immunohistochemistry with P-H3 specific antibody or in situ hybridization with c-fos or Perl probes.

peaking during the light period in the $\mathrm{SCN}^{12,13}$. As for c-fos, Per 1 expression is induced by light during the subjective night ${ }^{12,13}$. Daytime high levels of Perl were not blunted by baclofen (Fig. 4; CT3 and CT9), demonstrating that the effect of the drug is specific for the light-induced expression.

H3 phosphorylation closely followed c-fos and Per1 expression, as baclofen powerfully reduced the light-induced effect (Figs. 4 and $5 b$ ). Some $\mathrm{P}-\mathrm{H} 3$ positive nuclei remained in the mediolateral part of the SCN, which is consistent with what we and others ${ }^{29}$ have observed for c-fos induced expression. These results strongly suggest that the same signaling routes within the SCN are involved in this chromatin modification as in IEG and clock gene expression.

\section{H3 phosphorylation in the supraoptic nucleus}

GABA mediates neurotransmission from the SCN to magnocellular neurons in other hypothalamic nuclei, such as the paraventricular nucleus and the supraoptic nucleus $(\mathrm{SON})^{34,35}$.

Fig. 5. Baclofen induces histone $\mathrm{H} 3$ phosphorylation and $\mathrm{c}$-fos expression in the supraoptic nuclei (SO N) in a light-independent manner. (a) Brain cryosections from mice treated as described in Fig. 4, at CT9 or CT21, were processed for either immunohistochemistry with P-H 3 specific antibody or in situ hybridization with the c-fos probe. Representative images of the SO N regions are shown. Similar results were obtained at CT3 and CT 15 (data not shown). (b) Q uantification of the P-H 3 signal in the SCN at CT9 and CT21, after saline or baclofen injection, followed by a light pulse (light) or not (basal). The data shown are a compilation of several independent experiments, as indicated. In the SC N, the increase in P-H3 signal upon light stimulation at CT21, as well as the inhibition of this response by baclofen, are statistically significant (Student's t-test, $p<0.01$ ). In the SO N , the increase in P-H 3 signal upon treatment with baclofen is statistically significant (Student's t-test, $\mathrm{p}<0.001$ ).

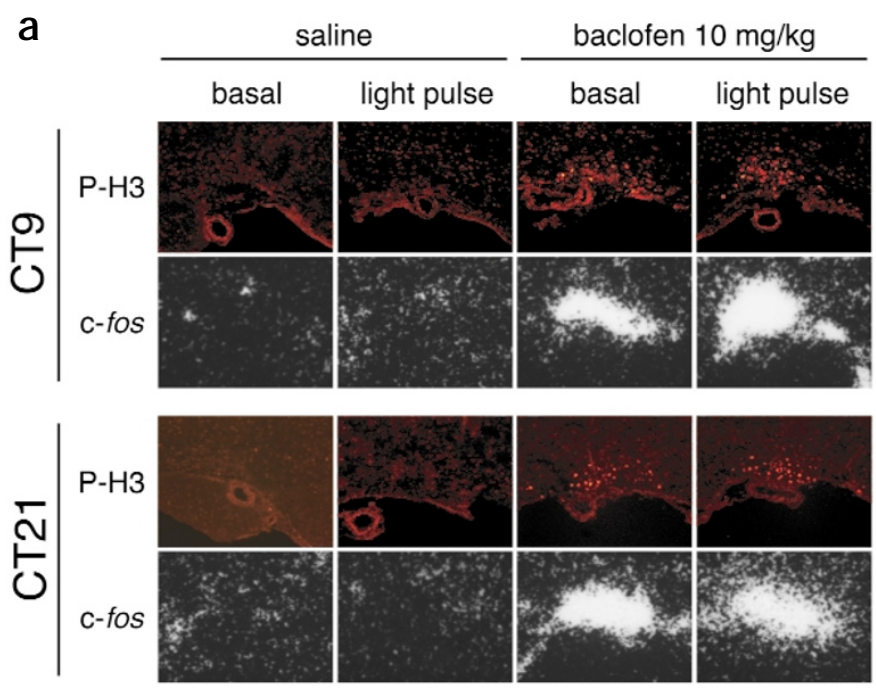

b

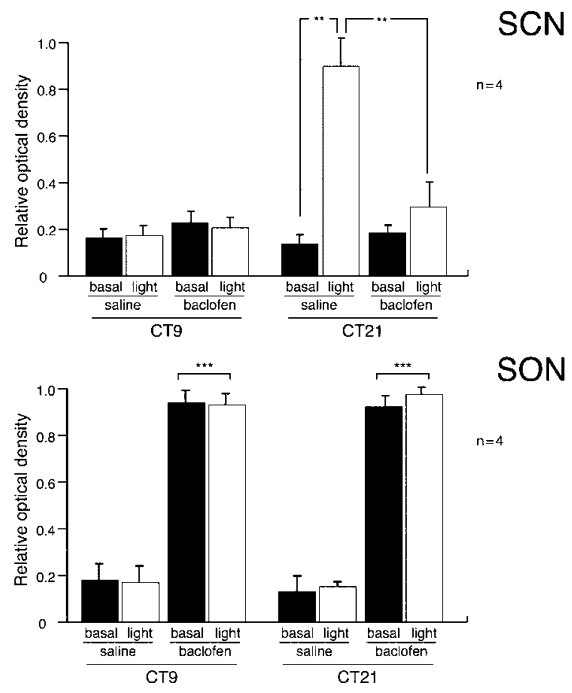


Moreover, various treatments induce Fos protein expression in the $\mathrm{SON}^{36,37}$. We asked whether induction of $\mathrm{H} 3$ phosphorylation accompanies c-fos gene activation in other neuronal settings. Thus, we explored the effect of baclofen administration and found that it triggered c-fos gene inducibility in cells of the SON (Fig. 5a). This induction was concomitant with a robust induction of H3 phosphorylation. Importantly, the baclofen-dependent induction of both c-fos gene expression and $\mathrm{H} 3$ phosphorylation was not clock regulated; it was observed at any time of the day. Moreover, the two regulatory events in the SON were independent of the light effect. Thus, although regulation was very different in the SON compared to the SCN (Fig. 5b), c-fos expression and $\mathrm{H} 3$ phosphorylation were still tightly coupled. These observations provide another independent and strong physiological link between these molecular events.

\section{Discussion}

Gene regulation requires condensation and decondensation of chromatin $^{23,24}$. One key step in this process is regulation of histone modifications, of which acetylation and phosphorylation have been the most studied cases ${ }^{23}$. Clock function relies greatly on transcriptional regulation. The generation of physiological circadian rhythms involves transcriptional regulatory feedback loops $s^{2,3}$, and one initial step in the integration of environmental stimuli is the rapid induction of a panel of genes ${ }^{12-15}$. Here, phase-shift-inducing light stimulation profoundly affected SCN cell physiology by triggering a major histone modification, possibly a primary event of the transcriptional cascade leading to regulated clock function.

Light treatment of mice during the subjective night elicits powerful induction of immediate-early genes, an event that seems to be important for clock phase-shifting ${ }^{16,17,38}$, although a more complex scenario is possible ${ }^{39,40}$. The data presented here provide evidence for a tight link between this transcriptional event and $\mathrm{H} 3$ phosphorylation in the SCN. First, the accumulation of c-fos mRNA and of $\mathrm{P}-\mathrm{H} 3$ present strikingly overlapping kinetics. Second, both events occur in the same region of the SCN, mainly in the ventrolateral region. Third, a high proportion of SCN neurons show colocalization of $\beta$-galactosidase and $\mathrm{P}-\mathrm{H} 3$ signal in light-treated fos-lac $Z$ transgenic mice. Fourth, induction of c-fos (and Per1) and H3 phosphorylation in reponse to light are severely blunted upon treatment with baclofen. These notions suggest that $\mathrm{H} 3$ phosphorylation is involved in the transcriptional response to light in the SCN, and therefore significantly participates in the phase-shift events induced by this stimulus. However, additional experiments are needed to causally link $\mathrm{H} 3$ phosphorylation to these processes. Previous studies in EGF-stimulated fibroblasts indicated that the same signal transduction pathway leads to a concerted phosphorylation of CREB and the $\mathrm{H} 3$ tail $^{25,27,41}$. A parallel with the SCN is interesting, as CREB phosphorylation is also induced in response to light ${ }^{19}$. The panel of genes activated by light in the SCN is likely to be different because $\mathrm{P}-\mathrm{H} 3$ labeling covers large patches throughout the nucleus, which contrast with the speckles observed in fibroblasts upon EGF stimulation ${ }^{25}$ (Fig. 1c).

Using c-fos-lacZ transgenic mice, we showed that $\mathrm{H} 3$ phosphorylation and c-fos promoter induction occurred in the same SCN neurons (Fig. 3c and d). However, some uncoupling was present. The most likely explanation was that scoring by immunoreaction only partially reflected the overlapping of the two events. Indeed, whereas the $\mathrm{P}-\mathrm{H} 3$ antibody revealed an early response event, the anti- $\beta$-gal signal was the result of accumulated $\beta$-gal protein generated with slower kinetics. Thus, many $\beta$-gal-positive nuclei were not $\mathrm{P}-\mathrm{H} 3$ positive, because the phosphorylation signal was long gone while the highly stable $\beta$-gal protein was still immunoreactive.

Induced $\mathrm{H} 3$ phosphorylation in the SCN and SON constituted a demonstration of non-mitotic $\mathrm{H} 3$ phosphorylation in vivo. The same modification was elicited, in each case, by very different stimuli. In the SCN, the light signal is transmitted through the RHT via the neurotransmitter glutamate and the ionotropic NMDA receptor ${ }^{6}$. In the SON, metabotropic GABA receptors are likely involved, because the response is stimulated by the $\mathrm{GABA}_{\mathrm{B}}$ receptor agonist baclofen. Given the distinct effectors used by these two types of receptors ${ }^{42,43}$, the intracellular pathways leading to $\mathrm{H} 3$ phosphorylation in vivo are likely to differ profoundly. Consistent with this notion is the observation that the mode of regulation in the two hypothalamic nuclei is distinct: in the SCN, H3 phosphorylation was light dependent, gated by the clock, and blocked by baclofen; in the SON, the response was equivalent at any time of the day, was independent of lighting conditions, and was induced by baclofen. The SON has an important involvement in osmoregulation, through osmosensitivity of its magnocellular neurosecretory cells and neurohypophysial hormone release ${ }^{44}$. H3 phosphorylation, and in general chromatin remodeling, may be involved in hormonal response governed by this physiological pathway.

In addition to counteracting the effect of light on IEG gene expression, baclofen blunts both the Per1 gene response and $\mathrm{H} 3$ phosphorylation. Several studies have addressed the question of the physiological role of GABA in the $\mathrm{SCN}^{45,46}$. GABA may be a coupling agent between $\mathrm{SCN}$ cells ${ }^{45}$, as it is able to acutely inhibit SCN neuron firing rate (through $\mathrm{GABA}_{\mathrm{B}}$ receptors) and shift the phase of these neurons (through $\mathrm{GABA}_{\mathrm{A}}$ receptors). In addition, it synchronizes SCN cells in culture when administered at regular times ${ }^{45}$. Finally, GABA seems to modulate gap junction communication between SCN neurons ${ }^{46}$. The involvement of additional chromatin modifications in a number of neuronal settings, and in response to a number of stimuli, is still to be explored.

Which H3 kinase is acting in response to light in the SCN? RSK2 is the H3 kinase in EGF-stimulated fibroblasts ${ }^{25}$. Other kinases, including MSK1 and PKA, have the capacity to phosphorylate $\mathrm{H} 3$ in vitro and are therefore among possible SCN candidates $^{26,47}$. All the above-mentioned kinases belong to the AGC group of protein kinases ${ }^{23}$. The search for the light-induced H3 kinase may reveal essential elements of the light transducing pathway and their links to central clock elements.

The molecular pathways by which light influences the circadian cycle are not fully recognized, although advances in various organisms implicate light-transducing molecules in clock function $^{48-50}$. Here we demonstrate that light acts directly on nuclear function by inducing a distinct histone modification, thus influencing the state of higher chromatin organization. This lightinduced chromatin modification is gated by the clock. Our results also demonstrate that multiple signaling pathways may converge to specific chromatin sites to elicit the same histone modification.

\section{Methods}

Animals and tissue preparation. We used wild-type 129/Sv mice, although similar results were obtained using other mouse strains. Trangenic mice (fos-lacZ) were obtained from T. Curran ${ }^{33}$. All animals were 7-12 week-old males. Mice were entrained on a L12-D12 (12 h light-12 h dark) for two weeks, and then placed in constant darkness (DD) for four days. They were then exposed to a 15-min light pulse and placed back in the dark for $15 \mathrm{~min}$ (unless otherwise mentioned). Baclofen $(10 \mathrm{mg} / \mathrm{kg})$, dissolved 
in $0.9 \% \mathrm{NaCl}$, or saline solution alone, was administered intraperitoneally $10 \mathrm{~min}$ before the light pulse. Mice were decapitated and dissected under dim red light conditions. Tissues to be assayed by western blot analysis were homogenized in boiling Laemmli buffer. For immunohistochemistry and in situ hybridization, tissues were placed in OCT and frozen on dry ice and $10-\mu \mathrm{m}$ thick coronal cryosections were prepared.

In situ hybridization. VIP (cloned by PCR from genomic mouse DNA, nucleotides 3095-3262 of the Genbank sequence X74297), c-fos and $m P e r 1$ (nucleotides 1-336 of ORF, Genbank sequence AF022992) [ $\left.{ }^{35} \mathrm{~S}\right]$-labeled antisense riboprobes were prepared by in vitro transcription (Promega, Madison, Wisconsin). As a control for nonspecific signal, consecutive sections were hybridized with c-fos, VIP and mPerl sense probes.

Immunohistochemistry. Brain sections were fixed in $1 \times$ PBS solution ( $\mathrm{pH} 7.5$ ) containing $4 \%$ paraformaldehyde, and then blocked for $1 \mathrm{~h}$ in $5 \%$ normal goat serum in $1 \times$ PBS $/ 0.05 \%$ Tween- 20 . For Ser- 10 -phosphorylated H3 (P-H3) immunolabeling, sections were incubated overnight at $4^{\circ} \mathrm{C}$ with a rabbit polyclonal antibody (1:1000 final dilution, Upstate Biotechnology, Lake Placid, New York). Slides were washed and incubated with a secondary antibody $\left(\mathrm{CY}^{\mathrm{TM}} 3\right.$ conjugated anti-rabbit serum, 1:1000; Jackson ImmunoResearch Laboratories, West Grove, Pennsylvania). Immunostained sections were counterstained with 4,6-diamidino-2phenylindole dihydrochloride (DAPI, Roche, Mannheim, Germany) before microscopy.

For double-labeling experiments, sections were incubated with a mouse monoclonal $\beta$-galactosidase-specific antibody (1:500, Sigma-Aldrich, St. Quentin Fallavier, France) and the P-H3 rabbit polyclonal antibody, and then incubated with a $\mathrm{CY}^{\mathrm{TM}_{3}}$ conjugated anti-mouse serum and a fluorescein (FITC) conjugated anti-rabbit serum (1:100 and 1:200 respectively, Jackson ImmunoResearch Laboratories).

We acquired images with DMLB Leica microscope with a HBO100W lamp. Confocal images were acquired on a Leica DMRE microscope. We analyzed retinal sections by the peroxidase-labeled detection method, and we introduced an additional step to block endogenous peroxidase activity.

Western blot analysis. Protein extracts were resolved by standard SDSPAGE. Samples were electroblotted onto Protan nitrocellulose (Schleicher \& Schuell, Dassel, Germany). Membranes were incubated in PBS/5\% low-fat milk and specific antibody for $12 \mathrm{~h}$ at $4^{\circ} \mathrm{C}$. Donkey, anti-rabbit HRP antibodies were used to reveal immunocomplexes by enhanced chemiluminescence (Pierce, Rockford, Illinois).

\section{ACKNOWLEDGEMENTS}

We thank T. Curran for the fos-lacZ transgenic mouse line. We thank $H$. Tei for the mPer1 cDNA, J. L. Vonesch for advice with confocal microscopy, E. Borrelli for reading the manuscript and $E$. Heitz and $M$. Rastegar for technical assistance. We thank Z. Travnickova, D. Whitmore, N. Foulkes, M. Pando and all members of the Sassone-Corsi laboratory for discussions and help. C.C. is supported by a postdoctoral fellowship from the European Community and N.C. by a Human Frontier Science Program long-term fellowship and a Canadian Institutes of Health Research postdoctoral fellowship. This work was supported by grants from Centre National de la Recherche Scientifique, Institut National de la Santé et de la Recherche Médicale, Centre Hospitalier Universitaire Régional, Fondation de la Recherche Médicale, Université Louis Pasteur, Human Frontier Science Program, Organon (Akzo/Nobel) and Association pour la Recherche sur le Cancer.

\section{RECEIVED 3 AUguST; ACCEPTED 18 OCTOBER 2000}

1. Klein, D., Moore, R. Y. \& Reppert, S. M. Suprachiasmatic Nucleus: The Mind's Clock (Oxford Univ. Press, New York, 1991).

2. Dunlap, J. C. Molecular bases for circadian clocks. Cell 96, 271-290 (1999).

3. Cermakian, N. \& Sassone-Corsi, P. Multilevel regulation of the circadian clock. Nat. Reviews Mol. Cell Biol. 1, 59-67 (2000).

4. Daan, S. \& Pittendrigh, C. S. A functional analysis of circadian pacemakers in nocturnal rodents. II. The variability of phase response curves. J. Comp. Physiol. 106, 253-266 (1976).
5. Moore, R. Y. \& Lenn, N. Y. A retinohypothalamic projection in the rat. J. Comp. Neurol. 146, 1-14 (1972).

6. Ding, J. M. et al. Resetting the biological clock: mediation of nocturnal circadian shifts by glutamate and NO. Science 266, 1713-1717 (1994).

7. Card, J. P. \& Moore, R. Y. Organization of lateral geniculate-hypothalamic connections in the rat. J. Comp. Neurol. 284, 135-147 (1989).

8. Moore, R. Y. \& Speh, J. C. GABA is the principal neurotransmitter of the circadian system. Neurosci. Lett. 150, 112-116 (1993).

9. Pickard, G. E. \& Rea, M. A. Serotonergic innervation of the hypothalamic suprachiasmatic nucleus and photic regulation of circadian rhythms. Biol. Cell 89, 513-523 (1997).

10. Johnson, R. F., Moore, R. Y. \& Morin, L. P. Loss of entrainment and anatomical plasticity after lesions of the hamster retinohypothalamic tract. Brain Res. 460, 297-313 (1988).

11. Harrington, M. E. \& Rusak, B. Lesions of the thalamic intergeniculate leaflet alter hamster circadian rhythms. J. Biol. Rhythms. 1, 309-325 (1986).

12. Albrecht, U., Sun, Z. S., Eichele, G. \& Lee, C. C. A differential response of two putative mammalian circadian regulators, mper 1 and mper2, to light. Cell 91, 1055-1064 (1997).

13. Shearman, L. P., Zylka, M. J., Weaver, D. R., Kolakowski, L. F. Jr. \& Reppert, S. M. Two period homologs: circadian expression and photic regulation in the suprachiasmatic nuclei. Neuron 19, 1261-1269 (1997).

14. Kornhauser, J. M., Nelson, D. E., Mayo, K. E. \& Takahashi, J. S. Photic and circadian regulation of c-fos gene expression in the hamster suprachiasmatic nucleus. Neuron 5, 127-134 (1990).

15. Morris, M. E., Viswanathan, N., Kuhlman, S., Davis, F. C. \& Weitz, C. J. A screen for genes induced in the suprachiasmatic nucleus by light. Science 279, 1544-1547 (1998).

16. Wollnik, F. et al. Block of c-Fos and JunB expression by antisense oligonucleotides inhibits light-induced phase shifts of the mammalian circadian clock. Eur. J. Neurosci. 7, 388-393 (1995).

17. Akiyama, M. et al. Inhibition of light- or glutamate-induced mPerl expression represses the phase shifts into the mouse circadian locomotor and suprachiasmatic firing rhythms. J. Neurosci. 19, 1115-1121 (1999).

18. Obrietan, K., Impey, S. \& Storm, D. R. Light and circadian rhythmicity regulate MAP kinase activation in the suprachiasmatic nuclei. Nat. Neurosci. 1, 693-700 (1998).

19. Ginty, D. D. et al. Regulation of CREB phosphorylation in the suprachiasmatic nucleus by light and a circadian clock. Science 260, 238-241 (1993).

20. De Cesare, D., Fimia, G. M. \& Sassone-Corsi, P. Signaling routes to CREM and CREB: plasticity in transcriptional activation. Trends Biochem. Sci. 24, 281-285 (1999).

21. Belvin, M. P., Zhou, H. \& Yin, J. C. The Drosophila $d C R E B 2$ gene affects the circadian clock. Neuron 22, 777-787 (1999).

22. Obrietan, K., Impey, S., Smith, D., Athos, J. \& Storm, D. R. Circadian regulation of cAMP response element-mediated gene expression in the suprachiasmatic nuclei. J. Biol. Chem. 274, 17748-17756 (1999).

23. Cheung, P., Allis, C. D. \& Sassone-Corsi, P. Signaling to chromatin through histone modifications. Cell 103, 263-271 (2000).

24. Workman, J. L. \& Kingston, R. E. Alteration of nucleosome structure as a mechanism of transcriptional regulation. Annu. Rev. Biochem. 67, 545-579 (1998).

25. Sassone-Corsi, P. et al. Requirement of Rsk-2 for epidermal growth factoractivated phosphorylation of histone H3. Science 285, 886-891 (1999).

26. Thomson, S. et al. The nucleosomal response associated with immediateearly gene induction is mediated via alternative MAP kinase cascades: MSK1 as a potential histone H3/HMG-14 kinase. EMBO J. 18, 4779-4793 (1999).

27. De Cesare, D., Jacquot, S., Hanauer, A. \& Sassone-Corsi, P. Rsk-2 activity is necessary for epidermal growth factor-induced phosphorylation of CREB protein and transcription of c-fos gene. Proc. Natl. Acad. Sci. USA 95, 12202-12207 (1998).

28. Cheung, P. et al. Synergistic coupling of histone $\mathrm{H} 3$ phosphorylation and acetylation in response to epidermal growth factor. Mol. Cell 5, 905-915 (2000).

29. Colwell, C. S., Kaufman, C. M. \& Menaker, M. Photic induction of Fos in the hamster suprachiasmatic nucleus is inhibited by baclofen but not by diazepam or bicucullin. Neurosci. Lett. 163, 177-181 (1993).

30. Gillespie, C. F. et al. GABAergic regulation of light-induced c-Fos immunoreactivity within the suprachiasmatic nucleus. J. Comp. Neurol. 411, 683-692 (1999).

31. Hendzel, M. J. et al. Mitosis-specific phosphorylation of histone $\mathrm{H} 3$ initiates primarily within pericentromeric heterochromatin during G2 and spreads in an ordered fashion coincident with mitotic chromosome condensation. Chromosoma 106, 348-360 (1997).

32. Silver, R. et al. Multiple regulatory elements result in regional specificity in circadian rhythms of neuropeptide expression in mouse SCN. Neuroreport 10, 3165-3174 (1999).

33. Robertson, L. M. et al. Regulation of $c$-fos expression in transgenic mice requires multiple interdependent transcription control elements. Neuron 14, 241-252 (1995).

34. Cui, L. N., Saeb-Parsy, K. \& Dyball, R. E. Neurones in the supraoptic nucleus of the rat are regulated by a projection from the suprachiasmatic nucleus. J. Physiol. (Lond.) 502, 149-159 (1997).

35. Cui, L. N., Coderre, E. \& Renaud, L. P. GABA(B) presynaptically modulates suprachiasmatic input to hypothalamic paraventricular magnocellular neurons. Am. J. Physiol. 278, R1210-1216 (2000). 
36. Wang, K., Guldenaar, S. E. \& McCabe, J. T. Fos and Jun expression in rat supraoptic nucleus neurons after acute vs. repeated osmotic stimulation. Brain Res. 746, 117-125 (1997).

37. Berquin, P., Bodineau, L., Gros, F. \& Larnicol, N. Brainstem and hypothalamic areas involved in respiratory chemoreflexes: a Fos study in adult rats. Brain Res. 857, 30-40 (2000).

38. Honrado, G. I. et al. The circadian system of $c$-fos deficient mice. J. Comp. Physiol. A 178, 563-570 (1996).

39. Colwell, C. S., Kaufman, C. M. \& Menaker, M. Phase-shifting mechanisms in the mammalian circadian system: new light on the carbachol paradox. J. Neurosci. 13, 1454-1459 (1993).

40. Weber, E. T., Gannon, R. L., Michel, A. M., Gillette, M. U. \& Rea, M. A. Nitric oxide synthase inhibitor blocks light-induced phase shifts of the circadian activity rhythm, but not c-fos expression in the suprachiasmatic nucleus of the Syrian hamster. Brain Res. 692, 137-142 (1995).

41. Xing, J., Ginty, D. D. \& Greenberg, M. E. Coupling of the RAS-MAPK pathway to gene activation by RSK2, a growth factor-regulated CREB kinase. Science 273, 959-963 (1996).

42. Hollmann, M. \& Heinemann, S. Cloned glutamate receptors. Annu. Rev.
Neurosci. 17, 31-108 (1994)

43. Bormann, J. The 'ABC' of GABA receptors. Trends Pharmacol. Sci. 21, 16-19 (2000).

44. Bourque, C. W. \& Oliet, S. H. Osmoreceptors in the central nervous system. Annu. Rev. Physiol. 59, 601-619 (1997).

45. Liu, C. \& Reppert, S. M. GABA synchronizes clock cells within the suprachiasmatic circadian clock. Neuron 25, 123-128 (2000).

46. Shinohara, K., Hiruma, H., Funabashi, T. \& Kimura, F. GABAergic modulation of gap junction communication in slice cultures of the rat suprachiasmatic nucleus. Neuroscience 96, 591-596 (2000).

47. Shibata, K., Inagaki, M. \& Ajiro, K. Mitosis-specific histone H3 phosphorylation in vitro in nucleosome structures. Eur. J. Biochem. 192, 87-93 (1990).

48. Ceriani, M. F. et al. Light-dependent sequestration of TIMELESS by CRYPTOCHROME. Science 285, 553-556 (1999).

49. van der Horst, G. T. et al. Mammalian Cry1 and Cry2 are essential for maintenance of circadian rhythms. Nature 398, 627-630 (1999).

50. Whitmore, D., Foulkes, N. S. \& Sassone-Corsi, P. Light acts directly on organs and cells in culture to set the vertebrate circadian clock. Nature 404, 87-91 (2000). 